\title{
CONTROL STUDY OF MULTI-MACHINE POWER SYSTEMS UNDER VARIATIONS OF MECHANICAL INPUT POWER AND COMMUNICATION DELAY
}

\author{
Igor Furtat \\ Institute for Problems of Mechanical Engineering, \\ Russian Academy of Sciences, Russia \\ cainenash@mail.ru
}

\section{Evgeny Tupichin}

Institute for Problems of Mechanical Engineering, Russian Academy of Sciences, Russia tupichin@mail.ru

\author{
Pavel Gushchin \\ Institute for Problems of Mechanical Engineering, \\ Gubkin Russian State University of Oil and Gas \\ (National Research University), Russia \\ guschin.p@mail.ru
}

\author{
Alexey Peregudin \\ Institute for Problems of Mechanical Engineering, \\ Russian Academy of Sciences, \\ ITMO University, Russia \\ zeekless@mail.ru
}

Article history:

Received 02.11.2019, Accepted 10.12.2019

\begin{abstract}
The paper studies the robust control for multi-machine power systems under parametric uncertainties, perturbed mechanical input power and unknown variable communication time-delay. Only relative speed of each electrical generator is available for measurement. The theoretical investigations show that the proposed algorithm synchronizes the multi-machine power system with the required accuracy in the normal mode and under symmetrical 3-phase short circuit faults which occur on transmission lines. Numerical investigations illustrate the efficiency of the proposed scheme for the three machines and the New England Power system benchmark.
\end{abstract}

\section{Key words}

Multi-machine power system, control, investigation.

\section{Introduction}

Currently there is a growing interest in control of multi-machine power systems especially in the context of Smart Grid [Butler, 2009; Farhangi, 2010; Liserre, Sauter, and Hung, 2010]. Since most components of power systems are electric generators, design of a simple and reliable controller for generators is important for quality control.

There exist a lot of methods for control of multimachine power systems. In [Qu et al., 1992] decentralized robust control algorithm for power systems is considered. Transient control of the sustained oscilla- tions that can occur after a major disturbance is investigated. The proposed control strategies are linear and require only local relative angle and velocity measurements for the model case, plus the measurement of mechanical power if turbine dynamics are included. The overall power system is shown to be exponentially stable in the large. The results are obtained without any linearization of the power system model.

In [Wang, Hill, and Guo, 1998; Guo, Hill, and Wang, 2000] the robust controller is designed for a multimachine power system modeled by differential-algebraic equations of the third order obtained in [Anderson and Fouad, 1977; Bergan, 1996]. It is assumed that parameters of power system are partially known and angle, relative speed, active electrical power and mechanical input power of each generator are available for measurement. The authors solve the problem in two steps. At the first step the authors use direct feedback linearization while at the second step they apply robust algorithm for control of linear model. Note that the control system of [Wang, Hill, and Guo, 1998; Guo, Hill, and Wang, 2000] may become unstable when faults (a symmetrical 3-phase short circuit fault which occur on one of the transmission lines) occur. The faults should be removed by opening the breakers of the fault lines.

In [Pogromsky, Fradkov, and Hill, 1996] adaptive synchronization algorithm is proposed for multi-machine power systems which models are described by the second order differential equations. Synthesis of control law is based on passivity and speed gradient method. 
The work [Ortega et al., 2005] is devoted to control of multi-machine power systems where generator models are presented by the third order differential-algebraic equations [Anderson and Fouad, 1977; Bergan, 1996]. The models of the load, the transmission lines and the infinite buses are described by the algebraic equations. The authors use the interconnection and damping assignment passivity-based control for synchronization of power system. Synthesized control algorithm requires measurements of the angles, relative speeds and transition EMF (electromotive force) directed along the transverse axis and parameters of the power system.

The paper [Mahmud, Pota, and Hossian, 2012] describes the nonlinear observer-based excitation controller for multi-machine power systems. It is assumed that only angle of each generator is available for measurement and parameters of power system are known. Exact feedback linearization is used to design the observer. The observed states of power system are directly used as the input to the controller where the control law does not need to be expressed in terms of all measured variables.

In [Leon, Mauricio, and Solsona, 2012] the nonlinear observer-based control for stabilization of power systems by using the excitation of synchronous generators is considered. The strategy goal is to attain maximum damping injection and to increase the transient stability, while good voltage regulation performance is maintained. For implementation of the control needs sensing currents and rotor speed, and knowledge of system parameters.

In [Benahdouga, Boukhetala, and Boudjema, 2012] the feedback linearization approach and high order sliding mode control are combined to stabilize and decentralize nonlinear multi-machine power systems. Each machine is modeled as an independent uncertain dynamic subsystem where the uncertainty is a disturbance that represents the effects of the rest of the system on that particular machine. A local high order sliding mode stabilizer is designed to regulate the machine angle of each generating unit under high level external disturbances.

Implementation of most existing algorithms [Qu et al., 1992; Wang, Hill, and Guo, 1998; Guo, Hill, and Wang, 2000; Anderson and Fouad, 1977; Bergan, 1996; Pogromsky, Fradkov, and Hill, 1996; Ortega et al., 2005; Mahmud, Pota, and Hossian, 2012; Leon, Mauricio, and Solsona, 2012; Benahdouga, Boukhetala, and Boudjema, 2012; Wenhua, Renjie, and Zhonghong, 1993; Tsykunov, 2007; Furtat and Fradkov, 2015; Furtat, Chugina, and Fradkov, 2015] requires measurement of the state vector for each generator and knowledge of the power system parameters. Besides the mechanical input power is constant and known and models of multimachine power systems [Qu et al., 1992; Wang, Hill, and Guo, 1998; Guo, Hill, and Wang, 2000; Anderson and Fouad, 1977; Bergan, 1996; Pogromsky, Fradkov, and Hill, 1996; Ortega et al., 2005; Mahmud, Pota, and Hossian, 2012; Leon, Mauricio, and Solsona, 2012; Be- nahdouga, Boukhetala, and Boudjema, 2012; Wenhua, Renjie, and Zhonghong, 1993; Tsykunov, 2007; Furtat and Fradkov, 2015; Furtat, Chugina, and Fradkov, 2015; Furtat, 2011; Fradkov and Furtat, 2013; Furtat et al., 2016; Furtat and Nekhoroshikh, 2018] do not take into account the possible presence of a communication time delay. Consider each problem in more detail.

Algorithms [Qu et al., 1992; Wang, Hill, and Guo, 1998; Guo, Hill, and Wang, 2000; Anderson and Fouad, 1977; Bergan, 1996; Pogromsky, Fradkov, and Hill, 1996; Ortega et al., 2005] require the measurement of the state vector of each generator such as angles and relative speed of the rotor generators, electrical power, transient EMF etc. However, the angle and the active electric power cannot be measured accurately at faults and, sometimes, in normal mode [Wang, Hill, and Guo, 1998; Guo, Hill, and Wang, 2000].

In [Qu et al., 1992; Wang, Hill, and Guo, 1998; Guo, Hill, and Wang, 2000; Anderson and Fouad, 1977; Bergan, 1996; Pogromsky, Fradkov, and Hill, 1996; Ortega et al., 2005; Mahmud, Pota, and Hossian, 2012; Leon, Mauricio, and Solsona, 2012; Benahdouga, Boukhetala, and Boudjema, 2012; Wenhua, Renjie, and Zhonghong, 1993] all the algorithms require knowledge of the power system parameters. For example, if the faults (symmetrical 3-phase short circuit faults) occur on transmission lines then these algorithms do not ensure the synchronization in the multi-machine power system. That is why these algorithms instant restore of transmission line with initial parameters is required.

Control systems in [Qu et al., 1992; Wang, Hill, and Guo, 1998; Bergan, 1996; Pogromsky, Fradkov, and Hill, 1996; Ortega et al., 2005; Mahmud, Pota, and Hossian, 2012; Leon, Mauricio, and Solsona, 2012] are designed under assumption of a constant mechanical input power. However, the synchronization loss may occur if mechanical input power is perturbed. Perturbed mechanical input power is a consequence of the mechanical torque ripple or regular oscillations of network loads. This situation usually occurs if the generators are driven by the piston engine [Wenhua, Renjie, and Zhonghong, 1993]. Moreover, we can observe low frequency lowdamped processes due to coincidence with the so-called reverse frequency of diesel and natural vibration frequency of the generator rotor.

Communication time-delays occur in a large geographic multi-machine power systems. Also, communication time-delays occur in information transmission channels, processing, measuring and controlling devices. In most cases this time delays are unknown and variable.

In the present paper we study the control system design for multi-machine power systems under parametric uncertainties, partially measured state vector of each generator, perturbed mechanical input power and unknown variable communication time-delay. For design the algorithm use the so-called method of auxiliary loop for compensation of unknown disturbances in power sys- 
tem. This method was first proposed for compensation of parametric uncertainties and external bounded disturbances in [Tsykunov, 2007]. The idea of this method is in the introduction of an auxiliary loop with desired parameters parallel to the plant. The difference between the output of the plant and the output of the auxiliary loop gives a function which depends on parametric and external disturbances. Then, this function is used for implementation of control law. Method of [Tsykunov, 2007] is applied to control of a network of electrical generators [Furtat and Fradkov, 2015] and a network of electrical generators with perturbed mechanical input power in [Furtat, Chugina, and Fradkov, 2015]. In [Furtat, 2011] the method of auxiliary loop is generalized for control of dynamical systems and in [Fradkov and Furtat, 2013] for control of dynamical networks with communication time delay.

In the present paper the results from [Furtat and Fradkov, 2015; Furtat, Chugina, and Fradkov, 2015; Furtat, 2011; Fradkov and Furtat, 2013; Furtat et al., 2016; Furtat and Nekhoroshikh, 2018] are generalized for control of multi-machine power systems with communication time delay. Moreover, the proposed algorithm is investigated on the New England Power system benchmark.

It is assumed that the relative speed of each generator is measured. The proposed algorithm provides synchronization of multi-machine power systems with the required accuracy in the normal mode and under symmetrical 3-phase short circuit faults which occur on transmission lines.

The paper is organized as follows. The problem statement is presented in Section 2. In Section 3 the application of the auxiliary loop method for control of power systems and the main result are considered. In Section 4 the efficiency of proposed scheme illustrated by a simulation of a power system consisting of three generators. Section 5 describes investigation of the proposed algorithm for New England Power system benchmark. Concluding remarks are given in Section 6. Appendix gives the proof of the auxiliary loop algorithm for control of power systems.

\section{Model of Multi-Machine Power Systems}

Consider the multi-machine power systems where ith subsystem is described by the following differentialalgebraic equations:

- Mechanical Dynamics:

$$
\begin{gathered}
\dot{\delta}_{i}(t)=\omega_{i}(t) \\
\dot{\omega}_{i}=-\frac{D_{i}}{2 H_{i}} w_{i}(t)-\frac{w_{0}}{2 H_{i}} \Delta P_{e i}, \quad i=1, \ldots, k
\end{gathered}
$$

— Electrical Dynamics:

$$
\dot{E}_{q i}^{\prime}(t)=\frac{1}{T_{d 0 i}^{\prime}}\left(E_{f i}(t)-E_{q i}(t)\right), \quad i=1, \ldots, k
$$

- Electrical Equations:

$$
\begin{gathered}
E_{q i}(t)=x_{a d i} I_{f i}(t)=E_{q i}^{\prime}(t)-\left(x_{d i}-x_{d i}^{\prime}\right) I_{d i}(t), \\
E_{f i}(t)=k_{c i} u_{f i}(t), \\
P_{e i}(t)=\sum_{j \in N_{i}} E_{q i}^{\prime}(t) E_{q j}^{\prime}(t) M_{i j} \sin \left(\delta_{i}(t)-\delta_{j}(t)\right), \\
Q_{e i}(t)=-\sum_{j \in N_{i}} E_{q i}^{\prime}(t) E_{q j}^{\prime}(t) M_{i j} \cos \left(\delta_{i}(t)-\delta_{j}(t)\right), \\
I_{d i}(t)=-\sum_{j \in N_{i}} E_{q j}^{\prime}(t) M_{i j} \cos \left(\delta_{i}(t)-\delta_{j}(t)\right), \\
I_{q i}(t)=\sum_{j \in N_{i}} E_{q j}^{\prime}(t) M_{i j} \sin \left(\delta_{i}(t)-\delta_{j}(t)\right), \\
V_{t i}(t)=\frac{1}{x_{d s i}} \sqrt{\left(E_{q i}^{\prime}(t)-x_{d i}^{\prime} I_{d i}(t)\right)^{2}+\left(x_{d i}^{\prime} I_{q i}(t)\right)^{2}}, \\
i=1, \ldots, k .
\end{gathered}
$$

Here $\delta_{i}(t)$ is an angle of the $i$-th generator with initial value $\delta_{i}(0)(\mathrm{rad}), \omega_{i}(t)$ is a relative speed $(\mathrm{rad} / \mathrm{s})$, $\omega_{0}$ is a synchronous machine speed $(\mathrm{rad} / \mathrm{s}), \Delta P_{e i}(t)=$ $P_{e i}(t)-P_{m i}(t), P_{e i}(t)$ is an electrical power, $P_{m i}(t)=$ $P_{m i 0}+\Delta P_{m i}(t), P_{m i 0}$ is a nominal mechanical input power, $\Delta P_{m i}(t)$ is a perturbation of mechanical input power, $D_{i}$ is a damping constant, $H_{i}$ is an inertia constant (s), $T_{d 0 i}^{\prime}$ is a direct axis transient short circuit time constant (s), $x_{d i}^{\prime}$ is a direct axis transient reactance, $x_{d i}$ is a direct axis reactance, $x_{a d i}$ is a mutual reactance between the excitation coil and the stator, $k_{c i}$ is a gain of the excitation amplifier, $u_{f i}(t)$ is an input of the SCR amplifier of the excitation loop, $E_{q i}(t)$ is an EMF in the quadrature axis, $E_{f i}(t)$ is an equivalent EMF in the excitation coil, $E_{q i}^{\prime}(t)$ is a transient EMF in quadratic axis, $I_{q i}(t)$ is a quadratic axis current, $I_{f i}(t)$ is an excitation current, $I_{d i}(t)$ is a direct axis current, $Q_{e i}(t)$ is a reactive power, $V_{t i}(t)$ is a $i$-th generator terminal voltage, $M_{i j}$ is an $i$-th row and $j$-th column element of nodal admittance matrix at the internal nodes after eliminating all physical buses, $N_{i}$ is a set of the adjacent generators for $i$-th generator. Assume that only parameters $D_{i}, H_{i}, T_{d 0 i}^{\prime}$, $x_{d i}^{\prime}, x_{d i}, x_{a d i}, x_{d s i}, k_{c i}, M_{i j}$ and perturbation $\Delta P_{m i}(t)$ are unknown and relative speeds $\omega_{i}(t), i=1, \ldots, k$ are available for measurement in the control system. Consider model (1)-(3) under the following assumptions.

\section{Assumptions}

1. Unknown parameters of model (1)-(3) belong to known bounded set $\Xi$. The perturbation $\Delta P_{m i}(t)$ and $\Delta \dot{P}_{m i}(t)$ are unknown bounded function.

2. The currents $I_{q i}(t), i=1, \ldots, k$ are available for measurement.

3. The faults are symmetrical 3-phase short circuit occurring on transmission lines.

The problem is to design a continuous control law $u_{f i}(t)$ defined as function of $\omega_{i}(t),\left.\omega_{j}(t)\right|_{j \in N_{i}}$ providing fulfillment of the following conditions

$$
\begin{aligned}
& \left|\delta_{i}(t)-\delta_{j}\left(t-\tau_{j i}(t)\right)\right|<\varepsilon_{1}, \\
& \left|\omega_{i}(t)-\omega_{j}\left(t-\tau_{j i}(t)\right)\right|<\varepsilon_{2},
\end{aligned}
$$


for $t>T$ and any value parameters of (1)-(3) from $\Xi$, where $\varepsilon_{1}>0, \varepsilon_{2}>0$ are prespecified sufficiently required accuracies, $\tau_{j i}(t)>0$ is an unknown communication time delay and $T>0$ is a transient time.

\section{Theoretical Investigation of Robust Algorithm for Multi-machine Power Systems}

Taking into account (3), rewrite (1) and (2) as follows

$$
\begin{gathered}
\dot{\delta}_{i}(t)=\omega_{i}(t), \\
\dot{\omega}_{i}(t)=-\frac{D_{i}}{2 H_{i}} \omega_{i}(t)-\frac{\omega_{0}}{2 H_{i}} \Delta P_{e i}(t), \\
\Delta \dot{P}_{e i}(t)=-\frac{1}{T_{d 0 i}^{\prime}} \Delta P_{e i}(t) \\
+\frac{1}{T_{d 0 i}^{\prime}} I_{q i}(t)\left[k_{c i} u_{f i}(t)+\left(x_{d i}-x_{d i}^{\prime}\right) I_{d i}(t)\right] \\
-\frac{1}{T_{d 0 i}^{\prime}} P_{m i}(t)+E_{q i}^{\prime}(t) \dot{I}_{q i}(t)-\Delta \dot{P}_{m i}(t) .
\end{gathered}
$$

Denote

$$
\begin{aligned}
& z_{i}(t)=\left[\delta_{i}(t), \omega_{i}(t), \Delta P_{e i}(t)\right]^{\mathrm{T}}, \\
& A_{i}=\left[\begin{array}{ccc}
0 & 1 & 0 \\
0 & -0.5 D_{i} H_{i}^{-1} & -0.5 \omega_{0} H_{i}^{-1} \\
0 & 0 & -\left(T_{d 0 i}^{\prime}\right)^{-1}
\end{array}\right], \\
& B_{i}(t)=\left[0,0, k_{c i} I_{q i}(t) / T_{d 0 i}^{\prime}\right]^{\mathrm{T}}, L=[0,1,0], \\
& f_{i}(t)=\frac{1}{k_{c i} I_{q i}(t)}\left[\left(x_{d i}-x_{d i}^{\prime} I_{q i}(t) I_{d i}(t)\right.\right. \\
& \left.+P_{m i}(t)+T_{d 0 i}^{\prime} E_{q i}^{\prime}(t) \dot{I}_{q i}(t)-T_{d 0 i}^{\prime} \Delta \dot{P}_{m i}(t)\right] .
\end{aligned}
$$

Rewrite equations (5) in the form

$$
\begin{gathered}
\dot{z}_{i}(t)=A_{i} z_{i}(t)+B_{i}(t)\left(u_{f i}(t)+f_{i}(t)\right), \\
\omega_{i}(t)=L z_{i}(t) .
\end{gathered}
$$

According to [Furtat and Fradkov, 2015; Furtat, Chugina, and Fradkov, 2015; Furtat, 2011; Fradkov and Furtat, 2013; Furtat et al., 2016], let us transform system of differential equation (6) to the differential equation in the following form

$$
Q_{i}(p) w_{i}(t)=R_{i}(p, t)\left(u_{f i}(t)+f_{i}(t)\right),
$$

where $Q_{i}(p), R_{i}(p, t)$ are linear differential operators getting from transformation from (6) to (7) at fixed time $t, \operatorname{deg} Q_{i}(p)=3, \operatorname{deg} R_{i}(p, t)=1, p=d / d t$.

Rewrite operators $R_{i}(p, t)$ and $Q_{i}(p)$ as follows [1620]

$$
\begin{aligned}
R_{i}(p, t) & =R_{0}(p)+\Delta R_{i}(p, t), \\
Q_{i}(p) & =Q_{0}(p)+\Delta Q_{i}(p) .
\end{aligned}
$$

where $R_{0}(p), Q_{0}(p)$ are linear differential operators chosen such that $\operatorname{deg} Q_{0}(p)=3, \operatorname{deg} R_{0}(p)=1$ and $Q_{0}(\lambda) / R_{0}(\lambda)=Q_{m}(\lambda), Q_{m}(\lambda)$ is any Hurwitz polynomial, $\lambda$ is a complex variable, $\Delta R_{i}(p, t)$ and $\Delta Q_{i}(p)$ are linear differential operators describing effects of uncertainties of (1)-(3). Substituting (8) in (7), rewrite equation (7) as follows

$$
Q_{m}(p) \omega_{i}(t)=u_{f i}(t)+\phi_{i}(t),
$$

where $R_{0}(p) \phi_{i}(t)=\Delta R_{i}(p, t) u_{f i}(t)-\Delta Q_{i}(p) \omega_{i}(t)+$ $R_{i}(p, t) f_{i}(t), \phi_{i}(t)$ is a function depending on uncertainties of $i$-th generator. Then the error equation $e_{i}(t)=$ $\sum_{j \in N_{i}}\left(\omega_{i}(t)-\omega_{j}\left(t-\tau_{j i}(t)\right)\right)$ can be rewritten in the form

$$
\begin{gathered}
Q_{m}(p) e_{i}(t)=\sum_{j \in N_{i}}\left(u_{f i}(t)-u_{f j}\left(t-\tau_{i j}(t)\right)\right. \\
\left.+\phi_{i}(t)-\phi_{j}\left(t-\tau_{i j}(t)\right)\right) .
\end{gathered}
$$

Adopting the approaches of [22], consider auxiliary loops with desired parameters in the following form

$$
Q_{m}(p) \bar{e}_{i}(t)=\chi u_{f i}(t), \quad i=1, \ldots, k .
$$

Here $\chi>0$ is a constant chosen by a designer. Taking into account (9) and (10), consider the mismatch function $\zeta_{i}(t)=e_{i}(t)-\bar{e}_{i}(t)$ obeying equation

$$
Q_{m}(p) \zeta_{i}(t)=\psi_{i}(t)
$$

where

$$
\begin{gathered}
\psi_{i}(t)=\sum_{j \in N_{i}}\left(u_{f i}(t)-u_{f j}\left(t-\tau_{i j}(t)\right)\right. \\
\left.+\phi_{i}(t)-\phi_{j}\left(t-\tau_{i j}(t)\right)\right)-\chi u_{f i}(t)
\end{gathered}
$$

is the function containing uncertainties of $i$-th generator, uncertainties of adjacent generators for $i$-th generator, uncertainties of generators communications and unknown communication time-delays.

Thus, auxiliary loop (10) allows a designer to obtain functions $\zeta_{i}(t)$ which depend on power system uncertainties and to use these functions in the control algorithm.

Since relative speeds $\omega_{i}(t), i=1, \ldots, k$ are available for measurement introduce control laws of

$$
u_{f i}(t)=-\chi^{-1} Q_{m}(p) \bar{\zeta}_{i}(t), \quad i=1, \ldots, k,
$$

where $\bar{\zeta}_{i}(t)$ is an estimate of the signal $\zeta_{i}(t)$ obtained from observer

$$
\begin{gathered}
\dot{\xi}_{i}(t)=G_{0} \xi_{i}(t)+D_{0}\left(\bar{\zeta}_{i}(t)-\zeta_{i}(t)\right), \\
\bar{\zeta}_{i}(t)=L_{1} \xi_{i}(t),
\end{gathered}
$$

where $\xi_{i}(t) \in \mathbb{R}^{2}$ is an estimate of $\theta_{i}(t)=\left[\zeta_{i}(t), \dot{\zeta}_{i}(t)\right]^{\mathrm{T}}, \quad G_{0}=\left[\begin{array}{ll}0 & 1 \\ 0 & 0\end{array}\right], D_{0}=$ $-\left[d_{1} \mu^{-1}, d_{2} \mu^{-2}\right]^{\mathrm{T}}$ coefficients $d_{1}, d_{2}$ are chosen 
such that the matrix $G=G_{0}-D L_{1}, D=\left[d_{1}, d_{2}\right]^{\mathrm{T}}$ is Hurwitz, $L_{1}=[1,0], \mu>0$ is a sufficiently small parameter. Observer (12) is designed following [Atassi and Khalil, 1999].

According to [Furtat, Fradkov, and Tsykunov, 014], consider vector $\bar{\eta}_{i}(t)=T^{-1}\left(\xi_{i}(t)-\theta_{i}(t)\right), T=$ $\operatorname{diag}\{\mu, 1\}$ characterizing quality of observer estimation. Taking into account (12) and differentiating $\bar{\eta}_{i}(t)$ with respect to $t$, one has

$$
\begin{gathered}
\dot{\bar{\eta}}_{i}(t)=\mu^{-1} G \bar{\eta}_{i}(t)+b \zeta_{i}^{(2)}(t), \\
\bar{\Delta}_{i}(t)=\mu L_{1} \bar{\eta}_{i}(t) .
\end{gathered}
$$

Here $b=[0,1]^{\mathrm{T}}$. Rewrite equations (13) as follows

$$
\begin{gathered}
\dot{\eta}_{i}(t)=\mu^{-1} G \eta_{i}(t)+\bar{b} \dot{\zeta}_{i}(t), \\
\bar{\Delta}_{i}(t)=\mu L_{1} \eta_{i}(t) .
\end{gathered}
$$

where $\bar{b}=[1,0]^{\mathrm{T}}$. Equations (12) and (13) are two different forms of the equation $\left(p^{2}+d_{1} \mu^{-1} p+\right.$ $\left.d_{2} \mu^{-2}\right) \bar{\eta}_{1 i}(t)=p^{2} \zeta_{i}(t)$. Taking into account (11) and (14), transform (9) to a state space form

$$
\begin{gathered}
\dot{x}_{i}(t)=A_{m} x_{i}(t)+\mu b g^{\mathrm{T}} \Delta_{i}(t), \\
e_{i}(t)=L_{1} x_{i}(t) .
\end{gathered}
$$

where $x_{i}(t) \in \mathbb{R}^{2}$ is a state vector of (15), $A_{m} \in \mathbb{R}^{2 \times 2}$ is matrix in Frobenius form with characteristic polynomial $Q_{m}(\lambda), \Delta_{i}(t)=\left[\eta_{1 i}(t), \dot{\eta}_{1 i}(t), \ddot{\eta}_{1 i}(t)\right]^{\mathrm{T}}, g$ is a vector composed of coefficients of $Q_{m}(\lambda)$.

The simplified block diagram of the control system for $i$-th generator is shown in Fig. 1 .

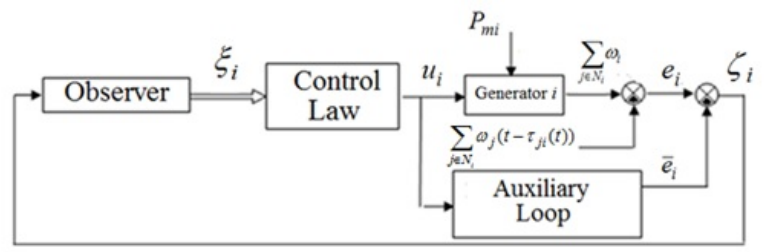

Figure 1. Block diagram of the control system for $i$-th generators

Theorem. Let assumptions 1-3 hold. Then there exist coefficients $\chi>0$ and $\mu_{0}>0$ such that for $0<\mu<$ $\mu_{0}$ control system (10)-(12) ensures the goal (4) and all signals in the closed-loop system are bounded.

The proof of the Theorem is given in Appendix.

Remark. It is seen from Theorem proof that the value $\varepsilon_{2}$ in (4) may be evaluated for $t=T$ as follows

$$
\varepsilon_{2}=\sqrt{\lambda_{\min }^{-1}(P)\left(V(0) e^{-\beta T}+\left(1-e^{-\beta T}\right) k \mu_{0} \bar{\psi}\right)},
$$

where $P$ is a the solution of the Lyapunov matrix equation

$$
A_{m}^{\mathrm{T}} P+P A_{m}=-Q_{1}, \quad Q_{1}=Q_{1}^{\mathrm{T}}>0,
$$

$\lambda_{\min }(\cdot)\left(\lambda_{\max }(\cdot)\right)$ is a minimum (maximum) eigenvalue of an appropriate matrix, $V(t)=V\left(x_{i}(t), \eta_{i}(t)\right)$ is Lyapunov function of the form

$$
V(t)=\sum_{i=1}^{k} x_{i}^{\mathrm{T}}(t) P x_{i}(t)+\sum_{i=1}^{k} \eta_{i}^{\mathrm{T}}(t) H \eta_{i}(t),
$$

$H$ is a the solution of the matrix equation

$$
G^{\mathrm{T}} H+G H=-Q_{2}, \quad Q_{2}=Q_{2}^{\mathrm{T}}>0,
$$

$$
\begin{gathered}
\beta=\min \left\{\lambda_{\max }^{-1}(P) \lambda_{\min }\left(Q_{3}\right), \mu_{0}^{-1} \lambda_{\max }^{-1}(H) \lambda_{\min }\left(Q_{4}\right)\right\}, \\
Q_{3}=Q_{1}-2 \mu_{0} P b g^{\mathrm{T}} g b^{\mathrm{T}} P, \quad Q_{4}=Q_{2}-2 H b b^{\mathrm{T}}, \\
\bar{\psi}=2 \sup _{t}\left\{\left|\Delta_{i}(t)\right|^{2}+\dot{\zeta}_{i}^{2}(t)\right\} .
\end{gathered}
$$

It follows from (16) that $\varepsilon_{2}$ explicitly depends on $\mu_{0}$. It is seen from (16) that decrease of $\mu_{0}$ in $\mathrm{n}$ times leads to decrease of $\varepsilon_{2}$ in (4) approximately in $\sqrt{n}$ times.

\section{Three-Machine Power Systems Control Study}

Consider multi-machine power system consisting of three electrical generators $G_{i}, i=1,2,3$ (Fig. 4), where third generator $G_{3}$ is a reference generator.

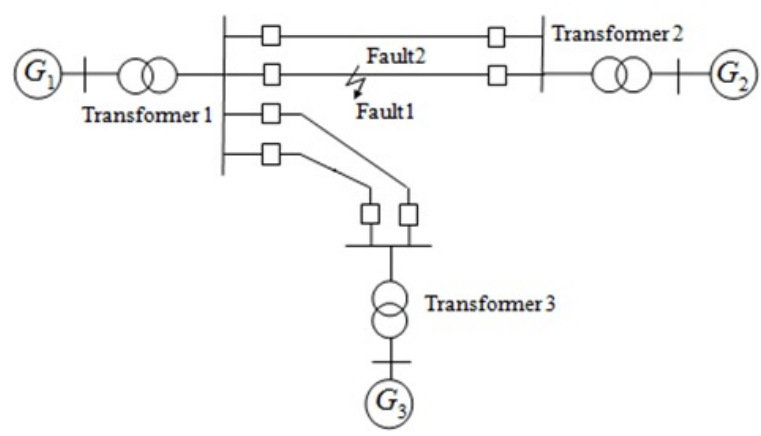

Figure 2. Multi-machine power system

Power system model is described by equations (1)(3). The set $\Xi$ of possible values of the model parameters (1)-(3) is defined by the following inequalities: $6 \leq T_{d 0 i}^{\prime} \leq 8,3 \leq D_{i} \leq 5,4 \leq H_{i} \leq 5.5,1 \leq k_{c i} \leq 3$, $1.8 \leq x_{d i} \leq 2.4,0.2 \leq x_{d i}^{\prime} \leq 0.4,0.3 \leq M_{i j} \leq 3$, $i, j=1,2,3$.

The auxiliary loop specifies transient performance of the closed-loop system. Choose $Q_{m}(p)=p^{2}+4 p+4$ and $\chi=1$. Then, (10) is as follows

$$
\left(p^{2}+4 p+4\right) \bar{e}_{i}(t)=-u_{f i}(t), \quad i=1,2,3 .
$$


Chose $D=[4,4]^{T}, \mu=0.01$ and rewrite observer (12) in the form

$$
\begin{gathered}
\dot{\xi}_{1 i}(t)=\xi_{2 i}(t)-4 \cdot 100\left(\xi_{1 i}(t)-\zeta_{i}(t)\right), \\
\dot{\xi}_{2 i}(t)=-4 \cdot 100^{2}\left(\xi_{1 i}(t)-\zeta_{i}(t)\right), \\
\xi_{1 i}(0)=\xi_{2 i}(0)=0,
\end{gathered}
$$

Define control laws (11) as

$$
u_{f i}(t)=\dot{\xi}_{2 i}(t)+4 \xi_{2 i}(t)+4 \xi_{1 i}(t), \quad i=1,2,3 .
$$

Let us consider two faults.

Fault 1 [Wang et al., 1998; Guo et al., 2000]. $M_{12}=$ $M_{21}=0.4853$ p.u. before time $t=1 \mathrm{~s} ; M_{12}=M_{21}=$ 2.9 at time $t=1 \mathrm{~s}$; the fault is removed $\left(M_{12}=M_{21}=\right.$ 0.4853 p.u.) by opening the breaker of the fault line at time $t=1.6 \mathrm{~s}$ (Fig. 4).

Fault 2. $M_{12}=M_{21}=0.4853$ p.u. before time $t=25$ s; $M_{12}=M_{21}=3$ at time $t=25 \mathrm{~s}$; the resistance of transmission line is equal to $M_{12}=M_{21}=0.8$ p.u. by opening the breaker of the fault line at time $t=25.6 \mathrm{~s}$ (Fig. 1).

Let parameters of generators $G_{i}, i=1,2,3$ be chosen as follows:

$G_{1}: D_{1}=4$ p.u., $H_{1}=5 \mathrm{~s}, T_{d 01}^{\prime}=1.5 \mathrm{~s}, x_{d 1}=1.8$ p.u., $x_{d 1}^{\prime}=0.257$ p.u., $P_{m 1}(t)=0.89+0.02 \sin (t)$ p.u., $V_{t 01}=1$ p.u., $k_{c 1}=1$ p.u., $\delta_{1}(0)=\pi / 3 \mathrm{rad}$;

$G_{2}: D_{2}=4.5$ p.u., $H_{2}=4.5 \mathrm{~s}, T_{d 02}^{\prime}=2.1 \mathrm{~s}$, $x_{d 2}=2.2$ p.u., $x_{d 2}^{\prime}=0.32$ p.u., $P_{m 2}(t)=0.83+$ $0.03 \sin (0.9 t)$ p.u., $V_{t 02}=0.9$ p.u., $k_{c 2}=1$ p.u., $\delta_{2}(0)=11 \pi / 36 \mathrm{rad}$;

$G_{3}: D_{3}=4.9$ p.u., $H_{3}=5.1 \mathrm{~s}, T_{d 03}^{\prime}=2 \mathrm{~s}, x_{d 3}=2$ p.u., $x_{d 3}^{\prime}=0.28$ p.u., $P_{m 3}(t)=0.85$ p.u., $V_{t 03}=1.1$ p.u., $k_{c 3}=1$ p.u., $\delta_{3}(0)=13 \pi / 36 \mathrm{rad}$;

$\omega_{0}=314.159 \mathrm{rad} / \mathrm{s}, \omega(0)=0 \mathrm{rad} / \mathrm{s}$ and $\Delta P_{e i}(0)=0$ p.u.

In addition consider power system with the following unknown communication time-delays:

$$
\begin{aligned}
& \tau_{31}(t)=0.01+0.01 \sin (t) \mathrm{s}, \\
& \tau_{12}(t)=\tau_{21}(t)=0.02+0.02 \cos (0.3 t) \mathrm{s} .
\end{aligned}
$$

Similarly to [Wang et al., 1998; Guo et al., 2000], introduce saturation of function $E_{f i}(t)$ such as $-3 \leq$ $E_{f i}(t) \leq 6$ p.u., $i=1,2,3$.

Fig. 3-6 show transients for generator angles $\delta_{i}(t)$, relative speeds $\omega_{i}(t)$ and signals $\Delta P_{e i}(t), i=1,2,3$. In Fig. 3-6 index "3" corresponds to reference generator, " $i-A L$ " corresponds to auxiliary loop algorithm for $i$-th generator, " $i-56$ " corresponds to algorithm [Wang et al., 1998; Guo et al., 2000] without perturbation in mechanical input power for $i$-th generator and " $i-56 p$ " corresponds to algorithm [Wang et al., 1998; Guo et al., 2000] when mechanical input power has perturbation for $i$-th generator. For example, in Fig. 3 the value $\delta_{3}$ corresponds to reference generator, $\delta_{1-A L}$ corresponds to auxiliary loop algorithm for the first generator, $\delta_{1-56}$ corresponds to algorithm [Wang et al., 1998; Guo et al., 2000] without perturbation in mechanical input power for the first generator, $\delta_{1-56 p}$ corresponds to algorithm [Wang et al., 1998; Guo et al., 2000] when mechanical input power has perturbation for the first generator.

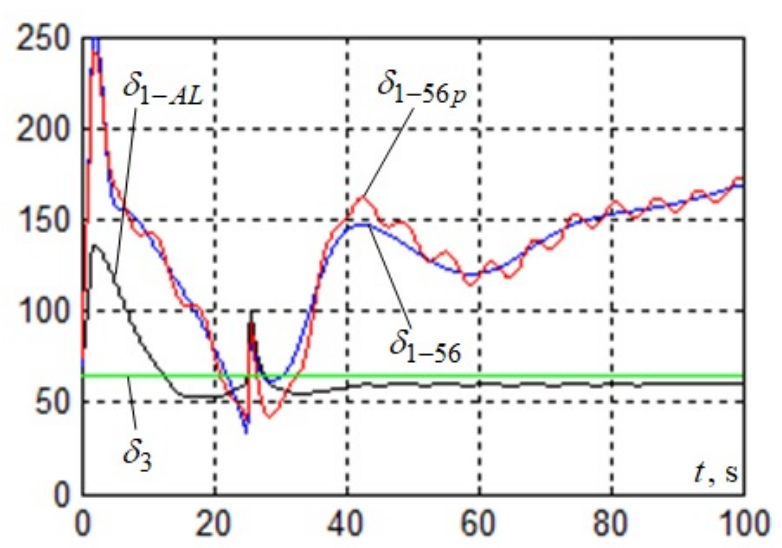

Figure 3. The transients of $\delta_{3}(t), \delta_{1-A L}(t), \delta_{1-56}(t)$, $\delta_{1-56 p}(t), \operatorname{rad}$.

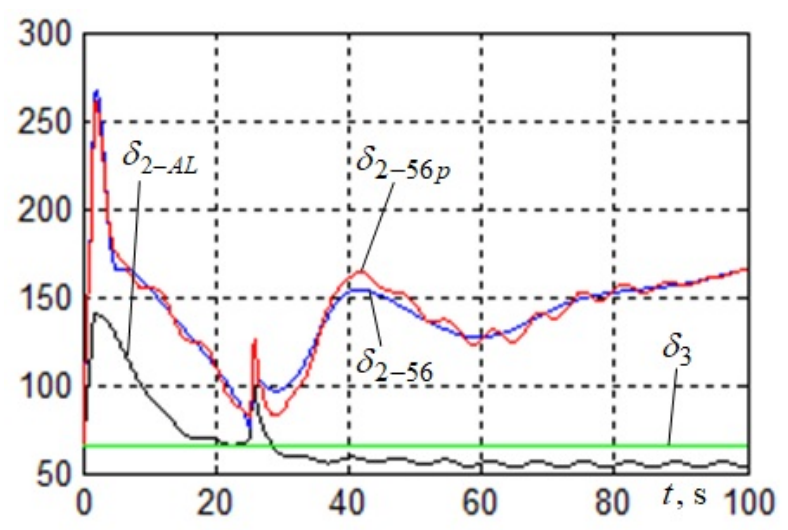

Figure 4. The transients of $\delta_{3}(t), \delta_{2-A L}(t), \delta_{2-56}(t)$, $\delta_{2-56 p}(t)$, rad.

The simulation results show that the designed algorithm ensures compensation of disturbances which depend on unknown parameters of multi-machine power system (which can be changed at faults) and perturbations in an input mechanical power when full state vector is not available to measurement. It follows from Fig. 3-6 that disturbances and influence of time delay are compensated by control system with the required accuracies $\varepsilon_{1}=2 \mathrm{rad}$ and $\varepsilon_{2}=0.1 \mathrm{rad} / \mathrm{s}$ achieved after $T=5 \mathrm{~s}$.

\section{New England Power System Benchmark Control Study}

Let us test algorithm (10)-(12) for the New England Power system benchmark consisting of 10 generators, 39 


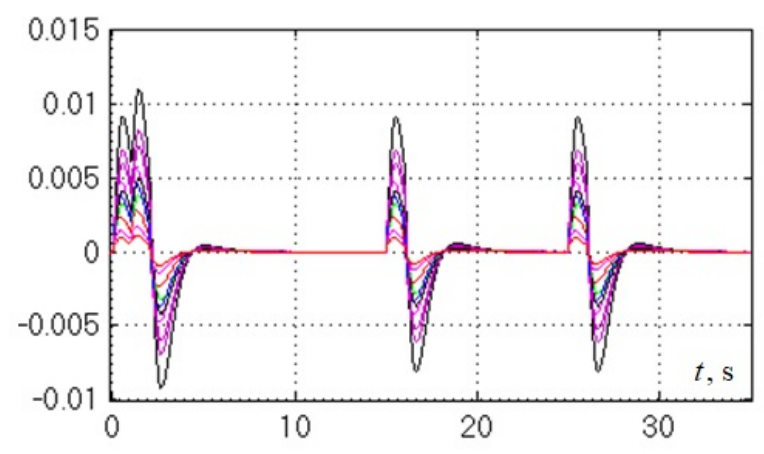

Figure 7. The transients of $\Delta \delta_{i}(t), i=1, \ldots, 10$, rad.

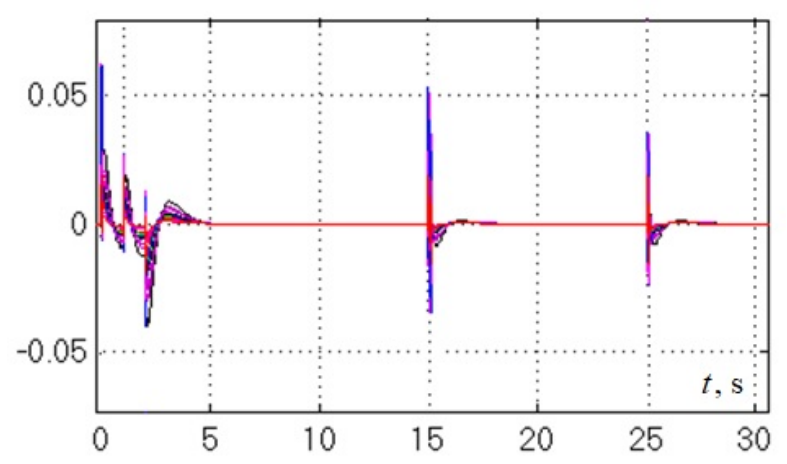

Figure 8. The transients of $\omega_{i}(t), i=1, \ldots, 10, \mathrm{rad} / \mathrm{s}$.

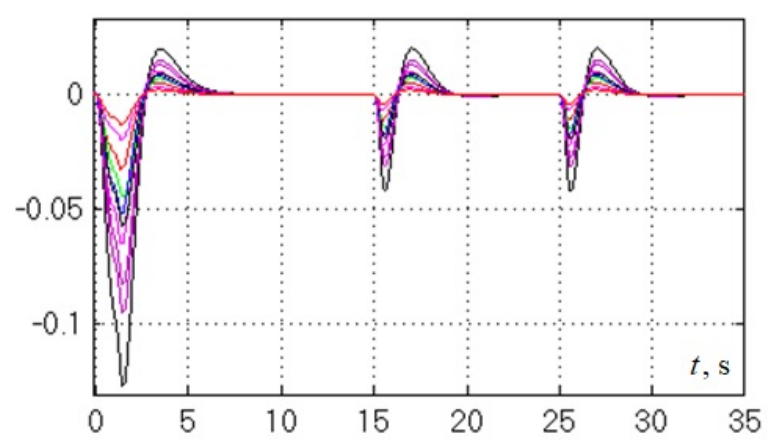

Figure 9. The transients of $\Delta V_{t i}(t), i=1, \ldots, 10, \mathrm{rad} / \mathrm{s}$.

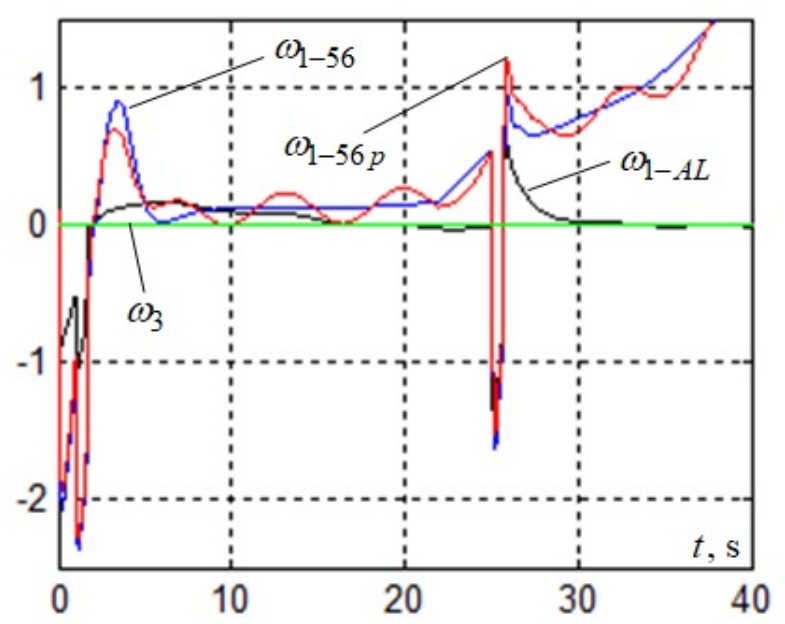

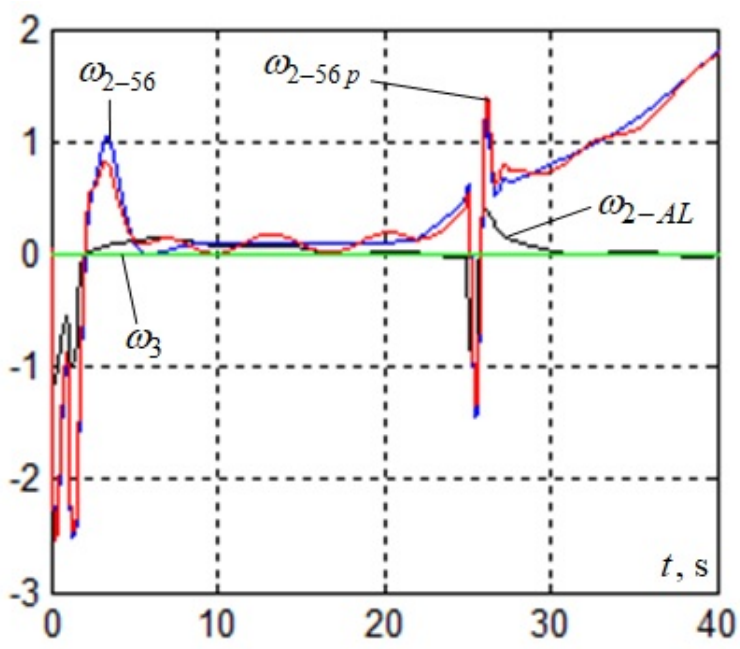

Figure 6. The transients of $\omega_{3}(t), \omega_{2-A L}(t), \omega_{2-56}(t)$, $\omega_{2-56 p}(t), \mathrm{rad} / \mathrm{s}$.

buses and 19 loads [Paul, 1992; The New England Power system benchmark].

To assess the controller robustness simulate three different faults. The first fault is 3-phase fault in bus number 16. It is introduced connecting a small impedance to ground at $t=1 \mathrm{~s}$ and cleared after $1 \mathrm{~s}$.

In the second fault the generator number 10 is suddenly disconnected from the network at $t=15 \mathrm{~s}$. This causes a significant lack of electrical power injected into the system.

The third fault consists in a permanent disconnection of the transmission line between buses 17 and 18 followed by a full load trip in bus 18 at $t=25 \mathrm{~s}$.

Fig. 7-9 show transients for the angle errors $\Delta \delta_{i}(t)=$ $\delta_{i}(t)-\delta_{i}(0)$, relative speeds $\omega_{i}(t)$ and voltage errors $\Delta V_{t i}(t)=V_{t i}(t)-V_{t i}(0), i=1, \ldots, 10$.

The simulation results show that the designed control system provides compensation of unknown parameters of the control system in normal mode and under faults.

The value $\varepsilon_{2}$ in goal (4) must be reduced by choice of $Q_{m}(p)$ and $\chi$ in auxiliary loop (10) and control law (11), and $\mu$ in observer (12).

Additional investigations under saturation of input signal and switched topology show that closed-loop system stable under the following restrictions $-2 \leq E_{f i}(t) \leq 2$ p.u., $i=1,2,3$, i.e. the control signal with small amplitude synchronizes the multi-machine power systems. 


\section{Conclusion}

In the present paper we study the proposed algorithm for control of multi-machine power systems with unknown parameters, perturbed mechanical input power and unknown variable communication time-delay. The algorithm provides synchronization of the power system and compensation of unknown parameters, disturbances and influence of time delay on stability of the closedloop system in normal mode and under faults, while multi-machine power systems without control my loss stability.

Simulation results for three generators exhibit better transients in the closed-loop system as compared with [Wang, Hill, and Guo, 1998; Guo, Hill, and Wang, 2000]. Moreover, unlike [Qu et al., 1992; Wang, Hill, and Guo, 1998; Guo, Hill, and Wang, 2000; Anderson and Fouad, 1977; Bergan, 1996; Pogromsky, Fradkov, and Hill, 1996; Ortega et al., 2005] the synthesis of the control algorithm does not require measurement the state vector of each generators and knowledge of power system parameters. Additionally, the proposed algorithm compensates the influence of communication time delay on stability of the closed-loop system. For control of power systems we need to measure only relative speed of each generator and to know the set of the possible values of power system parameters.

The proposed algorithm is studying on the New England Power system benchmark. The simulations show that the algorithm guarantees stability of the closed-loop system for the 3-phase fault, a fault caused by the generator suddenly disconnected from the network, and fault consisting in a permanent disconnection of the transmission line between buses.

\section{Acknowledgements}

The results of Sections III, IV, V and Appendix were developed under support of Russian Science Foundation (project no. 18-79-10104) in IPME RAS. The investigation under saturated control signal in Section $\mathrm{V}$ was partially supported by RFBR according to the research project No. 17-08-01266. The investigation under switched topology of network in Section V was partially supported by Program No. 30 of RAS Presidium.

\section{References}

Anderson, P. M. and Fouad, A. A. (1977). Power system control and stability. Iowa: Iowa State University Press.

Atassi, A. N. and Khalil, H. K. (1999). A separation principle for the stabilization of class of nonlinear systems. IEEE Trans. Automat. Control, 44(9), pp. 1672-1687.

Benahdouga, S., Boukhetala, D., and Boudjema, F. (2012). Decentralized high order sliding mode control of multi-machine power systems. Int J Electrical Power \& Energy Syst, 43, pp. 1081-1086.

Bergan, A. R. (1996). Power Systems Analysis. N .J.: Prentice-Hall.
Butler, F. (2009). A call to order a regulatory perspective on the smart grid. IEEE power \& energy magazine, pp. 16-93.

Farhangi, H. (2010). The path of the smart grid. IEEE power \& energy magazine, pp. 18-28.

Fradkov, A. L. and Furtat, I. B. (2013). Robust Control for a Network of Electric Power Generators. Automation and Remote Control, 74(11), pp. 1851-1862.

Furtat, I. and Nekhoroshikh, A. (2018). Investigation of electric generator robust algorithm under measurement noises. Cybernetics and Physics, 7(4), pp. 204-209.

Furtat, I. B. (2011). Robust control of electric generator with compensation of perturbations. Journal of Computer and Systems Sciences International, 50(5), pp. 785-792.

Furtat, I., Fradkov, A., and Tsykunov, A. (2014). Robust synchronization of linear dynamical systems with compensation of disturbances. Int J Robust and Nonlinear Control, 24(17), pp. 2774-2784.

Furtat, I. B. and Fradkov, A. L. (2015). Robust control of multi-machine power systems with compensation of disturbances. Int. Journal of Electrical Power \& Energy Systems, 73, pp. 584-590.

Furtat, I. B., Chugina, J. V., and Fradkov, A. L. (2015). Compensation of Disturbances in MultiMachine Power Systems Caused by Perturbation of Mechanical Input Power. Proc. of the 2015 European Control Conference (ECC 2015), Linz, Austria, pp. 794-799.

Furtat, I., Tergoev, N., Tomchina, O., Kazi, F., and Singh, N. (2016). Speed-Gradient-Based Control of Power Network: Case Study. Cybernetics and Physics, 5(3), pp. 85-90.

Guo, G., Hill, D. J., and Wang, Y. (2000). Nonlinear output stabilization control for multimachine power systems. IEEE Trans. Circuits Systems, 47(1), pp. 46-53.

Leon, A. E., Mauricio, J. M., and Solsona, J. A. (2012). Multi-machine power system stability improvement using an observer-based nonlinear controller. Electric Power Syst Research, 89, pp. 204-214.

Liserre, M., Sauter, T., and Hung, Y. J. (2010). Future energy systems. IEEE industrial electronics magazine, pp. 18-37.

Mahmud, M. A., Pota, H. R., and Hossian, M. J. (2012). Full-order nonlinear observer-based excitation controller design for interconnected power systems via exact linearization approach. Int J Electrical Power \& Energy Syst, 41, pp. 54-62.

Ortega, R., Galaz, M., Astolfi, A. Sun, Y., and Shen, T. (2005). Transient stabilization of multimachine power systems with nontrivial transfer conductance. IEEE Trans. Automat Control, 50(1), pp. 60-75.

Pai, M. A. (1989). Energy Function Analysis for Power System Stability. Kluwer Academic Publishers, Norwell, Massachusetts.

Pogromsky, A. Yu., Fradkov, A. L., and Hill, D. J. (1996). Passivity based damping of power system os- 
cillations. Proc. 35th IEEE Conference on Decision and Control, pp. 3876-3881.

Qu, Z., Dorsey, J. F., Bond, J., and McCalley, J. D. (1992). Application of robust control to sustained oscillation in power systems. IEEE Trans. Circuits and Systems - I: Fundament theory appl., 39(6), pp. 470476.

The New England Power system benchmark: http://www.mathworks.com/matlabcentral /fileexchange/54771-10-machine-new-englandpower-system-ieee-benchmark.

Tsykunov, A. M. (2007). Robust control algorithms with compensation of bounded perturbations. Automation and Remote Control, 71(7), pp. 1213-1224.

Wang, Y., Hill, D. J., and Guo, G. (1998). Robust decentralized control for multimachine power systems. IEEE Trans. Circuits and Systems-I: Fundament theory appl., 45(3), pp. 271-279.

Wenhua, L., Renjie, D., and Zhonghong, W. (1993). Integrated Optimal Control of Speed, Excitation and Load Sharing of Parallel Operating Diesel Generator Sets. Proc. 2nd IEE International Conference on Advances in Power System Control, Operation and Management, Hong Kong, 1, pp. 142-146.

\section{Appendix}

Proof of Theorem. Consider power systems in postfault-state. Transform equations (14) and (15) to the following system

$$
\begin{gathered}
\dot{x}_{i}(t)=A_{m} x_{i}(t)+\mu_{2} b g^{\mathrm{T}} \Delta_{i}(t), \\
\mu_{1} \dot{\eta}_{i}(t)+\mu_{2} b \dot{\zeta}_{i}(t) .
\end{gathered}
$$

To analyze system (20) the following Lemma [Furtat, Fradkov, and Tsykunov, 2014] is needed.

\section{Lemma.}

Let the system be described by the differential equation

$$
\dot{x}=f\left(x, \mu_{1}, \mu_{2}, t\right),
$$

where $x \in \mathbb{R}^{s_{1}}, \mu=\operatorname{col}\left(\mu_{1}, \mu_{2}\right) \in \mathbb{R}^{s_{2}}, f\left(x, \mu_{1}, \mu_{2}, t\right)$ is Lipchitz continuous function in $x$. Let (21) have a bounded closed set of attraction $\Omega=\{x \mid P(x) \leq C\}$ for $\mu_{2}=0$, where $P(x)$ is a piecewise-smooth, positive definite function in $\mathbb{R}^{s_{1}}$. In addition let there exist some numbers $C 1>0$ and $\bar{\mu}_{1}$ such that the following hold

$$
\begin{gathered}
\sup _{\left|\mu_{1}\right| \leq \bar{\mu}_{1}}\left[\left\langle\left[\frac{\partial P(x)}{\partial x}\right]^{\mathrm{T}}, f\left(x, \mu_{1}, 0, t\right)\right\rangle \mid P(x)=C\right] \\
\leq-C_{1} .
\end{gathered}
$$

Then there exists $\mu_{0}>0$ such that the system (21) has the same set of attraction $\Omega$ for $\mu_{2} \leq \mu_{0}$.
Let us check conditions of Lemma. Consider (20) for $\mu_{2}=0$ Then system (20) is asymptotically stable since $A_{m}$ and $G$ are Hurwitz matrixes. Therefore, functions $\Delta_{i}(t), \dot{\zeta}_{i}(t)$ are bounded. It follows from boundedness of $\Delta_{i}(t)$ and (12) that $\left|\xi_{i}(t)\right|<\infty$. In view of (11) control functions $u_{f i}(t)$ are bounded. Therefore, functions $u_{f j}\left(t-\tau_{j i}(t)\right), \Delta_{j}\left(t-\tau_{j i}(t)\right),\left|\xi_{i}\left(t-\tau_{j i}(t)\right)\right|$ and $\dot{\zeta}_{i}\left(t-\tau_{j i}(t)\right)$ are bounded. Therefore, according to Lemma there exists coefficient $\mu_{0}$ such that for $\mu_{1} \leq \mu_{0}$ and $\mu_{2} \leq \mu_{0}$ all functions are bounded in the closed-loop system. However, asymptotical stability of (20) does not follow for $\mu_{2}>0$ from asymptotical stability of the singularly perturbed system (20) for $\mu_{2}=0$. Therefore, consider (19) for $\mu_{1}=\mu_{2}=\mu_{0}$. Choose Lyapunov function $V\left(x_{i}, \eta_{i}\right)$ in the form (18). Differentiating (18) along the trajectories of (20), one has

$$
\begin{gathered}
\dot{V}(t)= \\
-\sum_{i=1}^{k} x_{i}^{\mathrm{T}}(t) Q_{1} x_{i}(t)+\sum_{i=1}^{k} 2 \mu_{0} x_{i}^{\mathrm{T}}(t) P b g^{\mathrm{T}} \Delta_{i}(t) \\
-\sum_{i=1}^{k} \mu_{0}^{-1} \eta_{i}^{\mathrm{T}}(t) Q_{2} \eta_{i}(t)+\sum_{i=1}^{k} 2 \eta_{i}^{\mathrm{T}}(t) H b \dot{\zeta}_{i}(t) .
\end{gathered}
$$

Find upper bounds for the second and the fourth terms of (22)

$$
\begin{aligned}
2 \mu_{0} x_{i}^{\mathrm{T}} P b g^{\mathrm{T}} \Delta_{i} & \leq 2 \mu_{0}\left[x_{i}^{\mathrm{T}} P b g^{\mathrm{T}} g b^{\mathrm{T}} P x_{i}+\left|\Delta_{i}\right|^{2}\right] \\
2 \eta_{i}^{\mathrm{T}} H b & \leq 2 \mu_{0}^{-1} H b b^{\mathrm{T}} \eta_{i}+2 \mu_{0} \dot{\zeta}_{i}^{2} .
\end{aligned}
$$

Then (22) can be rewritten as

$$
\dot{V} \leq \sum_{i=1}^{k}\left[-x_{i}^{\mathrm{T}} Q_{3} x_{i}-\mu_{0}^{-1} \eta_{i}^{\mathrm{T}} Q_{4} \eta_{i}\right]+\mu_{0} k \bar{\psi}
$$

Obviously, there exists $\mu_{0}>0$ such that $Q_{3}>0$ and $Q_{4}>0$. Taking into account (18), rewrite (23) as $\dot{V}(t) \leq-\beta V(t)+k \mu_{0} \bar{\psi}$. The solution of the last inequality satisfies

$$
V(t) \leq V(0) e^{-\beta t}+\left(1-e^{-\beta t}\right) k \mu_{0} \bar{\psi} .
$$

For $t=T$ the upper bound for $\left|\omega_{i}(t)-\omega_{j}(t)\right|$ is as follows

$$
\left|\omega_{i}(T)-\omega_{j}(T)\right| \leq\left|x_{i}(T)\right| \leq \sqrt{\lambda_{\min }^{-1}(P) V(T)} .
$$

Taking into account (25), obtain value (16) for $\varepsilon_{2}$ in (4). Theorem is proved. 